\title{
PKM2 Is Overexpressed in Glioma Tissues, and Its Inhibition Highly Increases Late Apoptosis in U87MG Cells With Low-density Specificity
}

\author{
JAE HYEON PARK*, JIN-SOL LEE*, YUNMOON OH, JI SUN LEE, HAE EUN PARK, \\ HAEUN LEE, YEON SU PARK, SO YOUNG KYUNG, HYUNG SIK KIM and SUNGPIL YOON
}

School of Pharmacy, Sungkyunkwan University, Suwon, Republic of Korea

\begin{abstract}
Background/Aim: Pyruvate kinase M2 (PKM2) functions as an important rate-limiting enzyme in aerobic glycolysis and is involved in tumor initiation and progression. However, there are few studies on the correlation between PKM2 expression and its role in glioma. Materials and Methods: PKM2 expression was immunohistochemically examined in human brain tumor samples. Furthermore, we studied the effects of two PKM2 inhibitors (shikonin and compound $3 K$ ) on the U87MG glioma cell line. Results: PKM2 was overexpressed in most glioma tissues when compared to controls. Interestingly, glioma-adjacent tissues from showed slight PKM2 overexpression. This suggests that PKM2 overexpression maybe an important trigger factor for glioma tumorigenesis. We found that the PKM2 inhibitor shikonin was effective against U87MG cells at a relatively low dose and was largely dependent on low cellular density compared to the effects of the anticancer drug vincristine. Shikonin highly increased late-apoptosis of U87MG cells. We also demonstrated that autophagy was involved in the increase in late-apoptosis levels caused by shikonin. Although vincristine treatment led to a high level of $G_{2}$-phase arrest in U87MG cells, shikonin did not increase $G_{2}$ arrest. Cotreatment with two PKM2 inhibitors, shikonin and compound $3 K$, increased the inhibitory effects. Conclusion: Combination therapy with PKM2 inhibitors together might be more effective than combination therapy with anticancer drugs.
\end{abstract}

This article is freely accessible online.

*These Authors contributed equally to this work.

Correspondence to: Sungpil Yoon, Ph.D., School of Pharmacy, Sungkyunkwan University, 2066 Seobu-ro, Jangan-gu, Suwon, Gyeonggi-do, 16419, Republic of Korea. Tel: +82 1055024893, Fax: +82 312928800, e-mail: syoon88@gmail.com

Key Words: PKM2, glioma, compound 3K, shikonin.
Our findings encourage the application of PKM2-targeting in gliomas, and lay the foundation for the development of PKM2 inhibitors as promising antitumor agents for glioma.

Malignant glioma is the most common and aggressive form of intrinsic brain tumor, and is accompanied by a very poor prognosis (1-3). The treatment of malignant glioma remains a worldwide challenge, although it has been studied for decades. Glioma therapies mainly consist of surgical excision combined with radiotherapy and chemotherapy (36). Patient survival rates are very low despite surgery in combination with postoperative chemotherapy and radiotherapy $(1,3,4,6,7)$. Thus, new therapies for treatment of glioma are urgently needed.

To meet the energy needs of unlimited proliferation, tumor cells increase their uptake of glucose and switch their energy source from mitochondrial oxidative phosphorylation to glycolysis, which is known as the Warburg effect $(8,9)$. During glycolysis, each glucose molecule is eventually converted into two molecules of pyruvate and two molecules of ATP in the cytoplasm (10-12). Pyruvate kinase M2 (PKM2) is a key rate-limiting enzyme of glycolysis in cancer cells. It has been demonstrated to contribute to the genesis and proliferation of glioma cells (13), and has thus recently gained much attention.

PKM2 is an alternatively spliced variant of the PKM gene, which is highly expressed in various cancer types and provides more selective growth advantages for tumor formation as compared to its counterpart PKM2 $(12,14,15)$. The overexpression of PKM2 results in increased glucose uptake, lactate production, and autophagy inhibition, thereby accelerating oncogenic growth $(9,10,12,16)$.

As triggering glycolysis dysfunction has emerged as a promising strategy for killing cancer cells, PKM2 has been considered as a targeting molecule. Various studies have used different strategies, involving the regulation of PKM2 expression, to determine the tumorigenic role of PKM2 in glioma and the therapeutic potential of PKM2-targeting 
Table I. Clinicopathological features of the tissue microarray samples.

\begin{tabular}{lcccc}
\hline Sample & N $(\%)$ & Tumor-adjacent tissue & Histological grade of tumor, $\mathrm{n}(\%)$ \\
\cline { 3 - 5 } & & & 2 & 3 \\
\hline Cancer patient & $24(100 \%)$ & $5(20.8 \%)$ & $9(37.5 \%)$ & $5(20.8 \%)$ \\
Healthy controls & $5(100 \%)$ & - & - & - \\
\hline
\end{tabular}

strategies $(17,18)$. However, the therapeutic role of PKM2 in gliomas remains unclear.

In this study, we investigated the correlation between glioma and PKM2 overexpression. We also assessed the anticancer effects of PKM2 inhibitors on human U87MG glioma cells. We hypothesized that PKM2-targeting may be a potential therapeutic strategy for gliomas.

\section{Materials and Methods}

Reagents and cell culture. Vincristine was obtained from Enzo Life Sciences (Farmingdale, NY, USA). PKM2 inhibitors, compound $3 \mathrm{~K}$ and shikonin, were purchased from SelleckChem (Houston, TX, USA).

The human brain carcinoma cell line U87MG was obtained from Dr. Suntaek Hong (Department of Health Sciences and Technology, GAIHST, Gachon University, Incheon, South Korea). Cells were grown in Dulbecco's modified Eagle's medium containing 10\% fetal bovine serum, $100 \mathrm{U} / \mathrm{ml}$ penicillin, and $100 \mathrm{mg} / \mathrm{ml}$ streptomycin (WelGENE, Daegu, Republic of Korea).

Brain tissue microarray and immunohistochemistry. Human tissue microarray slides (duplicate cores per case) of five normal brain tissues, five tumor-adjacent brain tissues, and 19 brain tumor tissues were obtained from US Biomax Inc. (Rockville, MD, USA). Detailed information regarding tissue microarray samples is presented in Table I. Immunohistochemical analysis was performed to investigate PKM2 expression in normal and brain tumor tissue samples as previously described (12). The tissue microarray slides were treated with xylene and ethanol, and then boiled in sodium citrate buffer. Tissue sections were treated with $5 \% \mathrm{H}_{2} \mathrm{O}_{2}$ for 15 $\min$ to inactivate endogenous peroxidases. Slides were incubated with PKM2 antibody (\#4053, 1:1000, dilution; Cell Signaling Technology, Danvers, MA, USA) at $4^{\circ} \mathrm{C}$ for $24 \mathrm{~h}$. The secondary anti-rabbit IgG (Vector Labs, Burlingame, CA, USA) was allowed to react at room temperature for $30 \mathrm{~min}$. Then horseradish peroxidase-conjugated streptavidin (Vector Labs) was added and allowed to react at room temperature for $30 \mathrm{~min}$. After staining with 3,3'-diaminobenzidine (Dako, Agilent, Santa Clara, CA, USA) and hematoxylin (Dako), then ethanol and xylene treatment, the slides were fixed on a cover glass using a mounting solution. Slides were then observed at $200 \times$ magnification using a confocal K1-fluo microscope (Nanoscope Systems, Daejeon, Republic of Korea).

For the evaluation of immunoreactivity and histological appearance, all tissue microarray slides were examined and scored by two pathologists. They screened the histological sections and selected areas of representative tumor cells, with one tissue core (2 $\mathrm{mm}$ in diameter) taken from each of the representative tumor samples and placed in a new recipient paraffin block. The intensity of PKM2 immunostaining in individual cells (average of duplicates) was scored in tissue according to the following scale, as previously established (12): 0 , No staining; 1 , weak intensity; 2 , moderate intensity; or 3 , strong intensity. The percentage of cells with immunostaining at each intensity was estimated, ranging from 0 to $100 \%$. The proportion of cells at each intensity level was multiplied by the corresponding intensity value, and these products were added to obtain an immunostaining score ranging from 0 to 300 .

Microscopic observation. Microscopic observations were performed as previously described (19-21), in order to examine the effect of shikonin, compound $3 \mathrm{~K}$, or vincristine on cellular growth. Briefly, U87MG cells with $30-40 \%$ confluence were treated with $5 \mathrm{nM}$ vincristine or $2 \mu \mathrm{M}$ shikonin for 1 or 2 days. For co-treatment experiments, U87MG cells with 30-40\% confluence were treated with $5 \mathrm{nM}$ vincristine, $1 \mu \mathrm{M}$ shikonin, 7.5 $\mu \mathrm{M}$ compound $3 \mathrm{~K}, 5 \mathrm{nM}$ VIC with $1 \mu \mathrm{M}$ shikonin, $5 \mathrm{nM}$ VIC with $7.5 \mu \mathrm{M}$ compound $3 \mathrm{~K}, 7.5 \mu \mathrm{M}$ compound $3 \mathrm{~K}$ with $1 \mu \mathrm{M}$ shikonin, or $0.1 \%$ DMSO for 1 day. Cells were then observed using an inverted microscope at $\times 40$ magnification.

The effects of these drugs at different cellular density were also examined. Briefly, U87MG cells were grown on $60 \mathrm{~mm}$-diameter dishes at low density $\left(2 \times 10^{5}\right.$ cells $)$ or high density $\left(4 \times 10^{5}\right.$ cells $)$. After 1 day, they were then treated with $5 \mathrm{nM}$ vincristine, $2 \mu \mathrm{M}$ shikonin, or $0.1 \%$ DMSO. The effect of these drugs on cellular growth was then examined after 1 day using an inverted microscope at $\times 40$ magnification.

Results were qualitatively confirmed in at least two independent experiments using an ECLIPSETs2 inverted microscope (Nikon, Tokyo, Japan) with a $40 \times$ or $100 \times$ objective lens (Nikon Microscopy U).

Fluorescence-activated cell sorting analysis. Fluorescenceactivated cell sorting analysis was performed as previously described (19-21), in order to determine whether PKM2 inhibitors led to cell-cycle arrest. Briefly, cells with $30-40 \%$ confluence were treated with $2 \mu \mathrm{M}$ shikonin, $15 \mu \mathrm{M}$ compound $3 \mathrm{~K}$, or $5 \mathrm{nM}$ vincristine for $24 \mathrm{~h}$. The cells were then treated with trypsin and collected by centrifugation. The pelleted cells were washed, suspended in $75 \%$ ethanol for at least $24 \mathrm{~h}$ at $-20^{\circ} \mathrm{C}$, washed with phosphate-buffered saline (PBS), and suspended in a propidium iodide and RNase A staining solution for $30 \mathrm{~min}$ at $37^{\circ} \mathrm{C}$. The cellcycle distribution of stained cells was qualitatively analyzed and the results confirmed in at least two independent experiments using a Guava EasyCyte Plus Flow Cytometer (Merck Millipore, Burlington, MA, USA). 
Annexin $V$ analysis. Annexin $\mathrm{V}$ analysis was performed using an annexin V-fluorescein isothiocyanate staining kit (BD Bioscience, Franklin, NJ, USA) as previously described (19-21), in order to measure apoptosis quantitatively. Briefly, cells with $30-40 \%$ confluence were treated with $2 \mu \mathrm{M}$ shikonin, $15 \mu \mathrm{M}$ compound $3 \mathrm{~K}$, or $5 \mathrm{nM}$ vincristine for $24 \mathrm{~h}$. The cells were then treated with trypsin and collected by centrifugation. The pelleted cells were washed, re-suspended with PBS. Cells with annexin V-fluorescein isothiocyanate and propidium iodide solution were then incubated for $30 \mathrm{~min}$ at $37^{\circ} \mathrm{C}$. The stained cells were qualitatively analyzed and the results confirmed in at least two independent experiments using a Guava EasyCyte Plus Flow Cytometer (Merck Millipore).

Cell viability assay. Colorimetric assay using the EZ-CyTox cell viability assay kit (Daeillab, Republic of Korea) was used for measuring cellular proliferation, as described previously (19-21). Briefly, cells with $30-40 \%$ confluence were grown in wells of 96well plates. The cells were then treated with $1-4 \mu \mathrm{M}$ of shikonin or $5 \mathrm{nM}$ vincristine for 1 or 2 days. EZ-CyTox solution was then added for $1 \mathrm{~h}$ at $37^{\circ} \mathrm{C}$. Absorbance at $450 \mathrm{~nm}$ was measured using the VERSA MAX Microplate Reader (Molecular Devices Corp. Sunnyvale, CA, USA). All experiments were performed at least in triplicate and repeated twice. The quantitative analysis was performed in at least two independent experiments in triplicate.

Acridine orange staining. Acridine orange staining was performed as described previously (12) in order to investigate whether autophagy was involved in the effects of PKM2 inhibitor. Cells were treated with $2 \mu \mathrm{M}$ of shikonin or $5 \mathrm{nM}$ vincristine for $24 \mathrm{~h}$. Cells were stained with acridine orange $(50 \mathrm{mg} / \mathrm{ml})$ by incubation for 15 min and then washed three times with Dulbecco's PBS to remove extracellular dye. Images were analyzed using a confocal K1-fluo microscope (Nanoscope Systems, Daejeon, Republic of Korea) at $40 \times$ magnification. Results were confirmed in at least two independent experiments.

Nuclear staining with 4',6-diamidino-2-phenylindole (DAPI). DAPI staining was used for observing apoptotic bodies, as previously described (12). Nuclear morphology and chromosomal condensation was also examined by DAPI staining. Cells grown in $60-\mathrm{mm}$ diameter dishes were treated with $2 \mu \mathrm{M}$ of shikonin or $5 \mathrm{nM}$ vincristine for $24 \mathrm{~h}$, and then fixed in acetone for $10 \mathrm{~min}$ at room temperature. Next, the cells were washed three times with PBS, stained with $0.1 \mu \mathrm{g} / \mathrm{ml}$ DAPI in PBS, and washed again with PBS. Apoptotic bodies and nuclear morphology were examined using fluorescence microscopy. Apoptotic cells were identified and determined based on characteristic observations, including the presence of condensed, fragmented, and degraded nuclei. Results were confirmed in at least two independent experiments.

Western blot analysis. Total cellular proteins were extracted as previously described $(12,22,23)$. Briefly, cells were treated with 2 $\mu \mathrm{M}$ of shikonin or $5 \mathrm{nM}$ vincristine for $24 \mathrm{~h}$, and then were washed with PBS and detached using trypsin. PRO-PREP ${ }^{\mathrm{TM}}$ protein extract solution (iNtRON, Seongnam, Republic of Korea) was used for isolating total proteins. A protein assay kit (Bio-Rad, Hercules, CA, USA) was used for measuring protein concentrations. The proteins were then subjected to western blot analysis as previously described $(23,24)$. Antibody against microtubule-associated proteins $1 \mathrm{~A} / 1 \mathrm{~B}$ light chain 3B (a-LC3B) was obtained from Cell Signaling
Technology (Danvers, MA, USA). Antibody against b-actin was obtained from Santa Cruz Biotechnology (Santa Cruz, CA, USA). Results were confirmed in at least two independent experiments.

Statistical analysis. All data are presented as the mean \pm S.D. of at least three independent experiments. Statistical analysis was performed using one-way analysis of variance, analysis of variance followed by Bonferroni's test. Analysis was performed using GraphPad Prism software (version 5.0; GraphPad Software, CA, USA). Statistical significance was set at $p<0.05$.

\section{Results}

PKM2 was overexpressed in glioma tissue compared with normal brain tissue. PKM2 expression was immunohistochemically examined in 19 human brain tumor tissues and five tumor-adjacent brain tissue samples obtained from patients. The patients' samples were compared with five normal human brain tissue samples obtained from healthy individuals (Table I). PKM2 expression was higher in most glioma tissues compared to that in normal brain tissues (Figure 1A and B), indicating that up-regulation of PKM2 is highly correlated with tumor progression in glioma. Most samples showed increased expression levels of PKM2 compared to controls, although there were some exceptions. There were no differences between males and females for PKM2 overexpression in glioma cells (data not shown). Interestingly, we found that the expression of PKM2 in tumor-adjacent normal tissues was slightly increased compared to that of tissues from controls but was not higher than that in glioma tissues. We assumed that adjacent glioma tissues have genetic alterations even if they have normal phenotypes. This suggests that PKM2 overexpression is an important factor in the development of tumorigenesis. It is possible that PKM2 can be a marker for glioma, and may represent a very early stage of cancer. A summary of the clinicopathological features of all tissues is provided in Table I. Collectively, we concluded that most glioma tissues had increased PKM2 expression compared to normal tissues.

A PKM2 inhibitor, shikonin, inhibits growth of U87MG glioma cell line at a low dose. Next, we aimed to identify novel mechanisms for PKM2 inhibition in glioma cells. We assume that our findings are more applicable for PKM2 inhibition in patients with brain cancer. We investigated the mechanisms of PKM2 inhibition in vitro using U87MG glioma cells (24). To inhibit PKM2 function, we used shikonin, a well-known PKM2 inhibitor (18, 25, 26). Vincristine was used as a positive control to compare the effectiveness of shikonin; at $5 \mathrm{nM}$, vincristine has been shown to effectively inhibit the growth of cancer cell lines from other organs (21). As seen in Figure 2A, the effect of $2 \mu \mathrm{M}$ of shikonin on $\mathrm{U} 87 \mathrm{MG}$ cell viability was similar to that of $5 \mathrm{nM}$ vincristine; thus, we concluded that even a low 
A

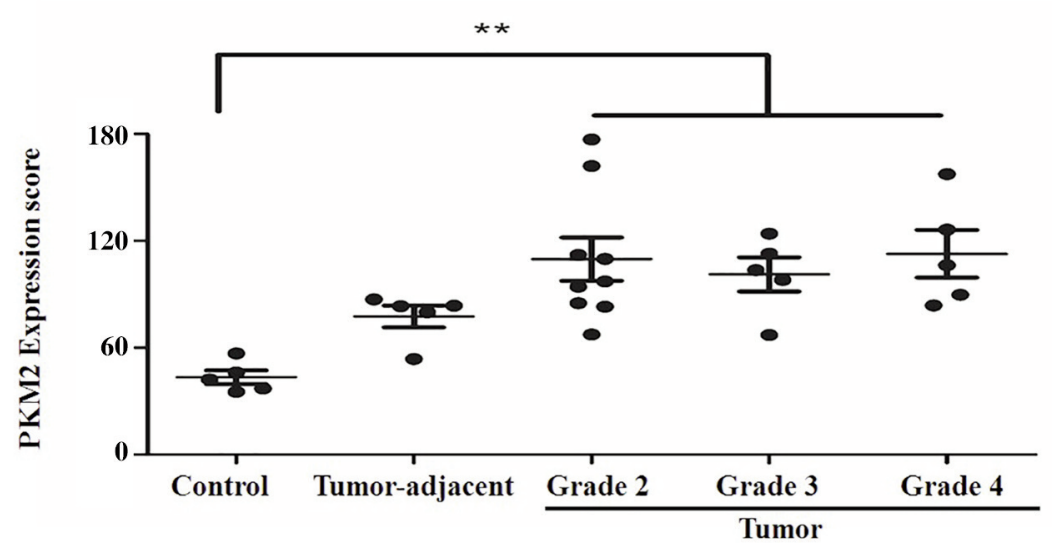

B

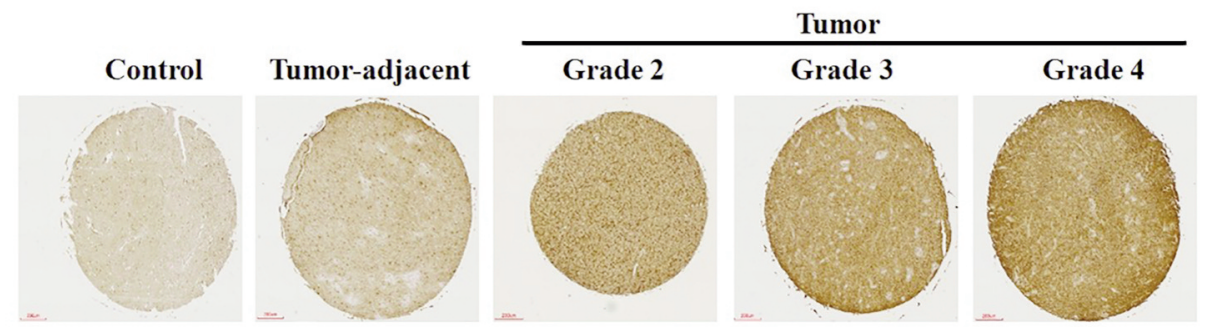

Figure 1. Pyruvate kinase M2 (PKM2) overexpression is observed in glioma tissues but not in normal brain tissues. A: Brain tumor, normal brain, and tumor-adjacent normal brain tissue samples were analyzed for changes in PKM 2 expression using immunohistochemistry. The intensity of PKM2 immunostaining in individual cells (average of duplicates) was scored in all tissue samples and in tumor according to grade. Scale bar=200 $\mu \mathrm{m}$. The values represent the mean $\pm S D$. **Significantly different at $p<0.01$. B: Photographs are shown of PKM 2 expression in representative tissues using immunohistochemistry.

dose of PKM2 inhibitor had a high effect. Since the effects of some drugs decline over time, we tested the effect of shikonin for $48 \mathrm{~h}$. As seen in Figure 2B, the effects of shikonin treatment for 24 and $48 \mathrm{~h}$ on cell viability were similar. We also confirmed our results using microscopic observations of U87MG cells (Figure 2C and D). These results indicated that at a relatively low dose, shikonin maintained its effects on U87MG cells over time.

Inhibition by shikonin highly depends on cellular density. The characteristics of drug effects on cancer cells can be determined by how much they depend on cellular density, since high cellular density inhibits drug effects (27). Therefore, we tested the extent to which the effect of shikonin depends on cellular density. As seen in Figure 2E and $\mathrm{F}$, we observed that under low-density culture of U87MG culture, vincristine and shikonin had similar inhibitory effects. However, when we increased cellular density, we found that shikonin did not inhibit U87MG cells to a greater extent than vincristine. Considering that vincristine has a similar effect on cells regardless of cell density, we concluded that shikonin, a PKM2 inhibitor, is a drug acting at low cell density. It is possible that PKM2 is not necessary for energy metabolism in high-density cellular states. We concluded that the effectiveness of PKM2 inhibition depended on the cellular density of glioma cells.

Shikonin highly increases the level of late-apoptosis. To further clarify the mechanism of action of shikonin, we performed quantitative apoptotic analysis using annexin $\mathrm{V}$ staining (12). As shown in Figure 3A, apoptotic cell death greatly increased after both shikonin and vincristine treatment. In a detailed analysis of apoptosis, the lateapoptosis level was increased two-fold over that of early apoptosis in shikonin treatment, whereas vincristine showed a smaller increase in late-apoptosis. This suggests that shikonin strongly induced apoptosis of U87MG cells. To evaluate late-apoptotic death after annexin V staining, we performed DAPI staining of U87MG cells. DAPI staining can detect late events during the apoptotic process. As seen in Figure 3B, we observed much more frequent chromosomal condensation in DAPI-stained U87MG cells with shikonin treatment than with vincristine treatment. This also supports the notion that shikonin is a strong and rapid inducer of late apoptosis. Collectively, we concluded that shikonin can induce rapid apoptosis in glioma cells. 

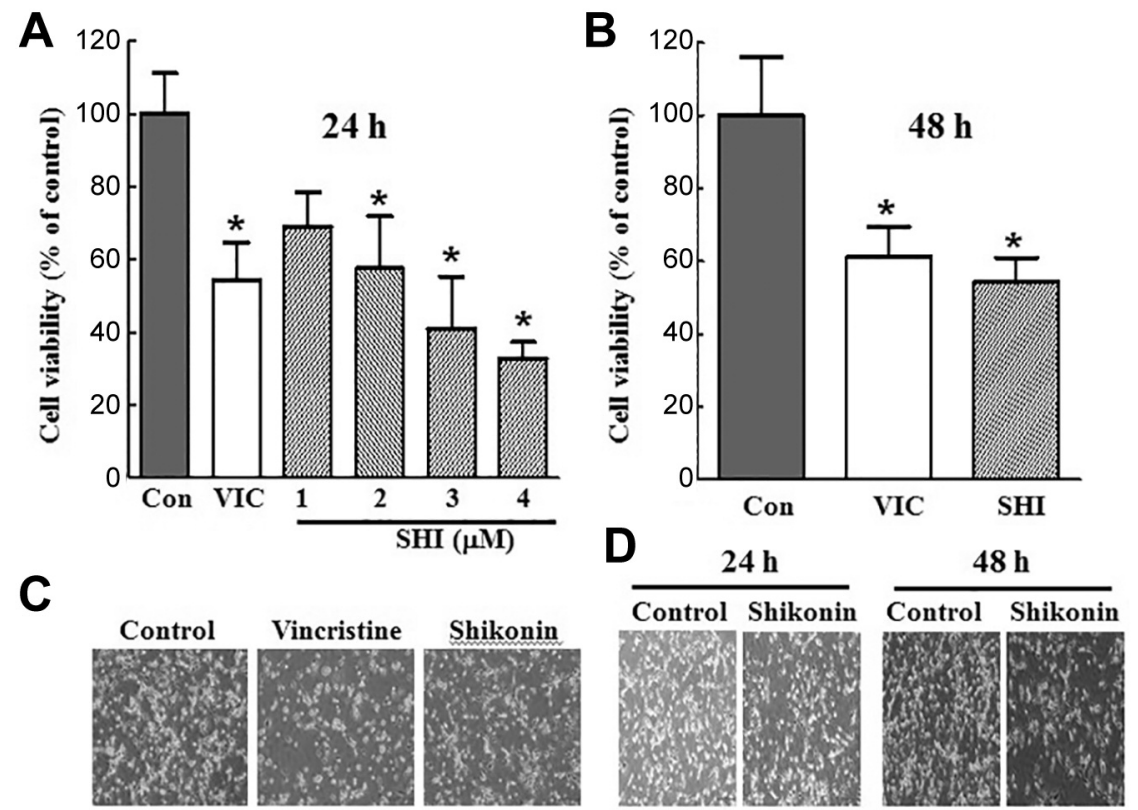

\section{E}
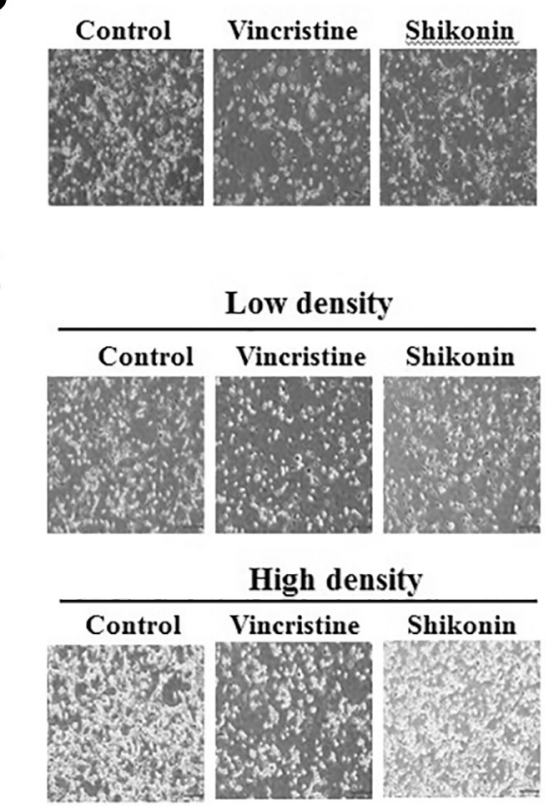

Figure 2. The pyruvate kinase M2 inhibitor shikonin inhibits growth of U87MG glioma cell line cultured at low density. A: U87MG glioma cells were plated on 96-well plates and grown to 30-40\% confluence. The cells were then stimulated for $24 \mathrm{~h}$ with $5 \mathrm{nM}$ vincristine (VIC), 1-4 $\mu \mathrm{M}$ shikonin (SHI), or $0.1 \%$ dimethyl sulfoxide (DMSO; (Con). Cell viability assay was performed. B: U87MG glioma cells were plated on 96-well plates and grown to 30-40\% confluence. The cells were then stimulated for $48 \mathrm{~h}$ with $5 \mathrm{nM}$ vincristine (VIC) or $2 \mu \mathrm{M}$ shikonin. The data are presented as the mean \pm S.D. of at least two experiments repeated in triplicate experiments. *Significantly different at p $<0.05$ compared to the corresponding control. C: U87MG cells were grown on $60 \mathrm{~mm}$-diameter dishes and treated with $5 \mathrm{nM}$ vincristine, $2 \mu \mathrm{M}$ shikonin, or $0.1 \%$ DMSO (Control). After 1 day, cells were observed using an inverted microscope at $\times 40$ magnification. D: U87MG cells were grown on 60 mm-diameter dishes and treated with $2 \mu \mathrm{M}$ shikonin, or $0.1 \%$ DMSO (Control). After $24 \mathrm{~h}$ or $48 \mathrm{~h}$, cells were observed using an inverted microscope at $\times 40$ magnification. E: U87MG cells were grown on 60 mm-diameter dishes either with low density $\left(2 \times 10^{5}\right.$ cells) or high density $\left(4 \times 10^{5}\right.$ cells $)$. They were then treated with 5 nM vincristine, $2 \mu M$ shikonin, or $0.1 \%$ DMSO (Control). After 1 day, all cells were observed using an inverted microscope at $\times 40$ magnification.

The PKM2 inhibitor does not increase $G_{2}$-phase arrest of U87MG cells. Next, we performed fluorescence-activated cell sorting analyses to determine whether cell-cycle arrest occurs together with apoptosis in shikonin-treated cells (20, 21). As shown in Figure $3 \mathrm{C}$, vincristine considerably increased the number of cells in $\mathrm{G}_{2}$-phase arrest compared to that in the control cells, in a dose-dependent manner. This indicates that the reduction in cellular viability in vincristinetreated $\mathrm{U} 87 \mathrm{MG}$ cells resulted from $\mathrm{G}_{2}$-phase cell-cycle arrest. However, we did not detect a significant increase in $\mathrm{G}_{2}$-phase arrest in shikonin-treated U87MG cells (Figure $3 \mathrm{C})$. Considering that shikonin led to a high rate of late apoptosis of U87MG cells (Figure 3A and B), we concluded that non- $\mathrm{G}_{2}$-phase arrest by shikonin stimulated the apoptotic 
A

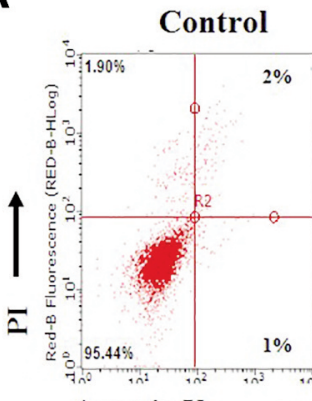

Annexin V
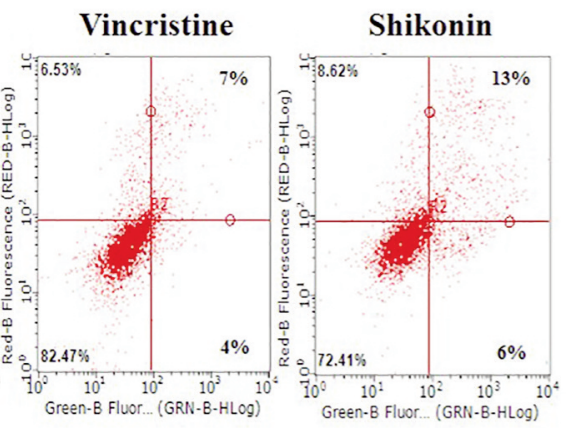

B

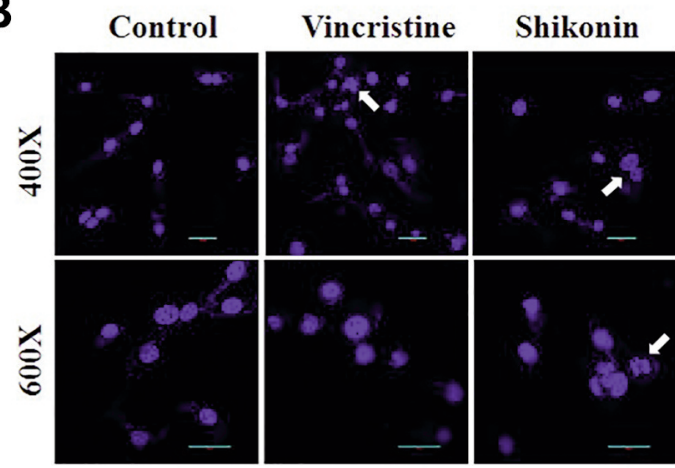

C
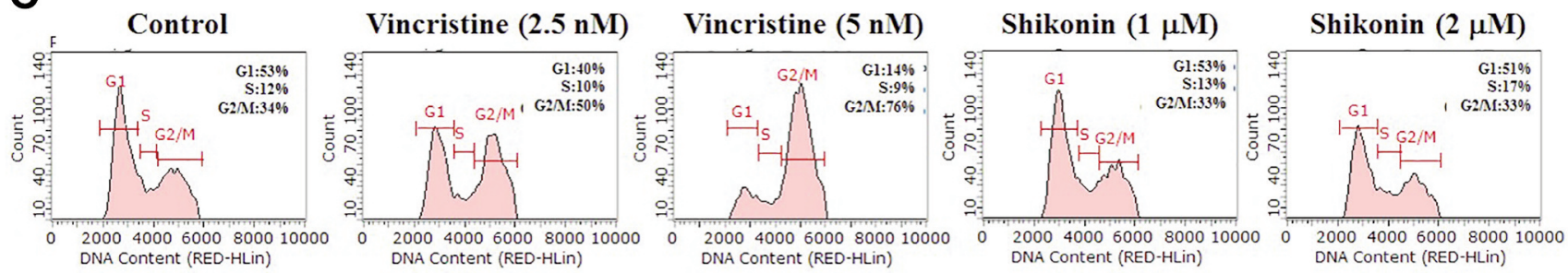

E
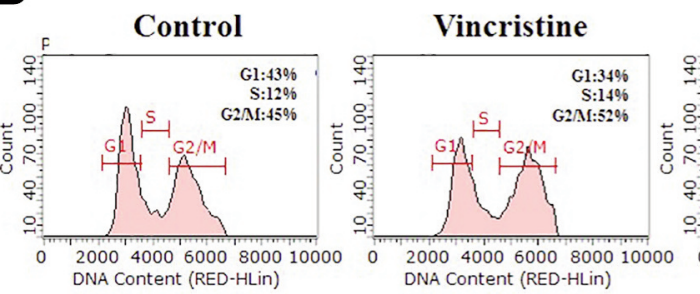

\section{Compound 3K}

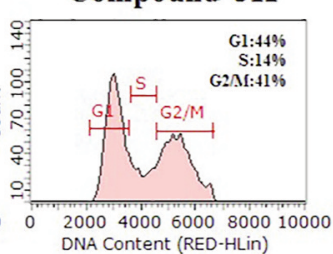

Control

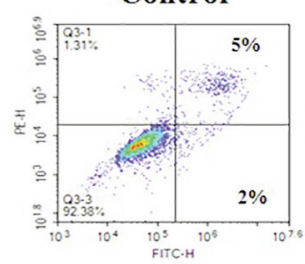

Compound $3 \mathrm{~K}$

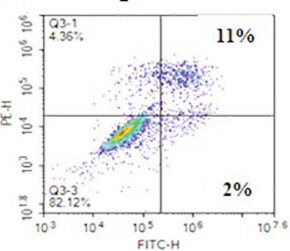

Figure 3. Shikonin strongly induces late apoptosis in U87MG cells. A: U87MG cells were grown on 60 mm-diameter dishes. They were then treated with $5 \mathrm{nM}$ vincristine, $2 \mu \mathrm{M}$ shikonin, or $0.1 \%$ dimethyl sulfoxide (DMSO; Control). After $24 \mathrm{~h}$, annexin V-propidium iodide (PI) analyses were performed. B: U87MG cells were grown on $60 \mathrm{~mm}$-diameter dishes. They were then treated with $5 \mathrm{nM}$ vincristine, $2 \mu \mathrm{M}$ shikonin, or $0.1 \%$ DMSO (Control). After 24 h, staining with 4',6-diamidino-2-phenylindole was performed. C: U87MG cells were grown on 60 mm-diameter dishes and treated with vincristine, shikonin or $0.1 \%$ DMSO (Control). After 24 h, fluorescence-activated sorting (FACS) analyses were performed. D: U87MG cells were grown on $60 \mathrm{~mm}$-diameter dishes and treated with $5 \mathrm{nM}$ vincristine, $15 \mu \mathrm{M}$ compound $3 \mathrm{~K}$, or $0.1 \%$ DMSO (Control). After $24 \mathrm{~h}$, FACS analyses were performed. E: U87MG cells were grown on $60 \mathrm{~mm}$-diameter dishes. They were then treated with $15 \mu \mathrm{M}$ compound $3 \mathrm{~K}$, or $0.1 \%$ DMSO (Control). After $24 \mathrm{~h}$, annexin V-PI analyses were performed.

pathway in U87MG glioma cells. In a detailed quantitative analysis, we also found that shikonin treatment increased Sphase arrest in a dose-dependent manner (Figure 3C). This suggested that DNA replication was prevented by shikonin treatment. We assumed that this S-phase increase also contributed to faster and stronger pathways of induction of late apoptosis.

Another PKM2 inhibitor, compound 3K, has a similar mechanism of inhibition to that of shikonin. We also tested whether PKM2 inhibitors have similar mechanisms in inhibiting U87MG glioma cells. Compound 3K was recently developed and is generally considered a PKM2-specific inhibitor $(12,28-31)$. As seen in Figure 3D and E, compound
3K highly increased late apoptosis in U87MG glioma cells without $\mathrm{G}_{2}$-phase arrest, whereas vincristine significantly increased $\mathrm{G}_{2}$-phase arrest. These results indicate that the two PKM2 inhibitors studied have have similar inhibitory mechanisms in glioma cells. Considering that $\mathrm{G}_{2}$-phase arrest is one of the survival mechanisms in cancer against toxic agents, we concluded that PKM2 inhibitors are highly effective targeting drugs for faster apoptotic death without allowing cellular survival delays in glioma cells.

Shikonin increases induction of autophagy in U87MG glioma cells. To further clarify the mechanism of late apoptotic death induced by shikonin, we studied autophagic cell death. PKM2 inhibits autophagy formation to increase cancer 

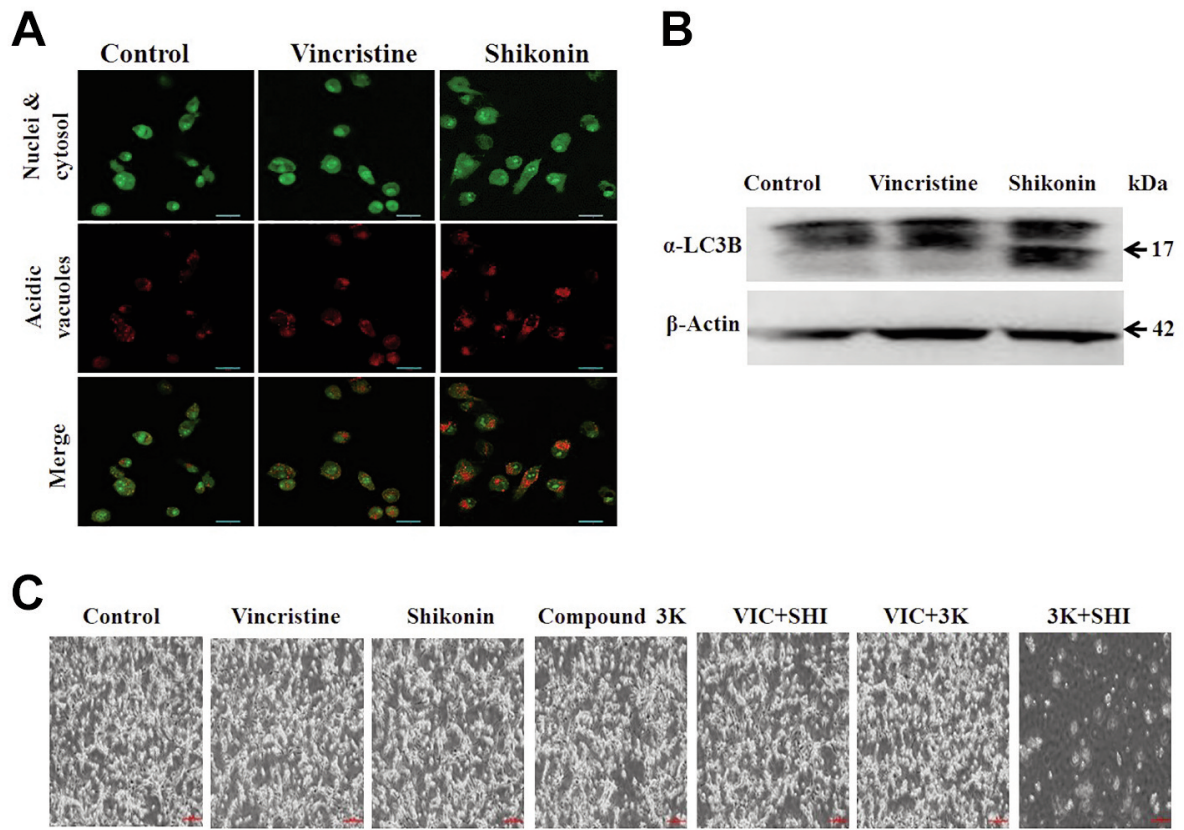

Figure 4. The combination of two different PKM2 inhibitors synergistically reduces growth of U87MG glioma cells. A: U87MG cells were grown on $60 \mathrm{~mm}$-diameter dishes. They were then treated with $5 \mathrm{nM}$ vincristine, $2 \mu \mathrm{M}$ shikonin, or $0.1 \%$ DMSO (Control). After 24 h, acridine orange staining was performed. B: U87MG cells were grown on $60 \mathrm{~mm}$-diameter dishes. They were then treated with $5 \mathrm{nM}$ vincristine, $2 \mu M$ shikonin, or $0.1 \%$ DMSO (Control). After $24 \mathrm{~h}$, western blot analysis was performed using antibodies against the marker of autophagosome formation microtubule-associated proteins $1 \mathrm{~A} / 1 \mathrm{~B}$ light chain $3 B(\alpha-L C 3 B)$ and b-actin. C: U87MG cells were grown on 60 mm-diameter dishes and treated with $5 \mathrm{nM}$ vincristine, $1 \mu \mathrm{M}$ shikonin, $7.5 \mu \mathrm{M}$ compound $3 \mathrm{~K}, 5 \mathrm{nM}$ VIC with $1 \mu \mathrm{M}$ shikonin $(V I C+S H I), 5 \mathrm{nM}$ VIC with $7.5 \mu \mathrm{M}$ compound $3 \mathrm{~K}$ $(V I C+3 K), 7.5 \mu M$ compound $3 K$ with $1 \mu M$ shikonin $(3 K+S H I)$, or $0.1 \%$ DMSO (Control). After 1 day, cells were observed using an inverted microscope at $\times 40$ magnification.

survival or proliferation. To identify autophagy induction by shikonin, acridine orange staining and western blot analysis were performed (12). As shown in Figure 4A, acridine orange staining was highly accumulated in acidic vacuoles in shikonin-treated U87MG cells, indicating that autophagy largely increased. However, vincristine-treated U87MG cells showed much less staining (Figure 4A), suggesting that with autophagy contributed less to late apoptosis induced by vincristine. These results indicate that the high level of lateapoptotic death caused by shikonin results from the induction of autophagic death of U87MG glioma cells. Increased autophagy induced by shikonin was also confirmed with degradation of a-LC3B protein, which is a major marker of autophagosome formation. As seen in Figure 4B, shikonin, but not vincristine, increased a-LC3B degradation, suggesting that shikonin induced autophagic death I of U87MG cells. Collectively, we concluded that shikonin induced high levels of late-apoptotic cell death via both non$\mathrm{G}_{2}$-phase arrest and induction of autophagy.

The combination of two different PKM2 inhibitors synergistically reduces growth of U87MG glioma cells. We hypothesized that co-treatment with an anticancer drug (vincristine) and a PKM2 inhibitor (shikonin or compound $3 \mathrm{~K}$ ) would have additive effects in U87MG glioma cells, since they have different mechanisms action. As seen in Figure 4C, however, we did not observe increased inhibition in co-treatments with vincristine with shikonin nor with compound $3 \mathrm{~K}$ when compared to single treatments. Interestingly, we found that co-treatment of shikonin with compound $3 \mathrm{~K}$ led to greater growth-inhibitory effects when compared to single treatments (Figure 4C). These results suggest that different types of PKM2 inhibitors can be used for combination therapy against glioma.

\section{Discussion}

The role of PKM2 has been studied in tumor initiation and progression in various cancer types $(9,10,12,16)$. It has been established that inhibition of PKM2 reduces tumor growth and causes cancer cell death. Studies have revealed the overexpression of PKM 2 mRNA in various human cancer type, including liver, bladder, breast, lung, esophageal, gastric, and colorectal $(8,10,14,15)$. Furthermore, the overexpression of the PKM2 protein has been associated with different types of human cancer. Since there are few 
studies on the correlation between PKM2 and its role in glioma $(11,32,33)$, we studied the relationship between PKM2 and glioma. Although previous studies have shown the effects of PKM2 inhibition of glioma cells $(13,17,18$, 34 ), we attempted to identify the mechanism of PKM2 inhibition. We also anticipated that our novel findings about the role of PKM2 in glioma might facilitate the application of PKM2-targeting in glioma in clinical applications.

Firstly, our immunohistochemical analysis revealed that PKM2 levels were higher in most patients with glioma than those in heathy controls. This suggested that the upregulation of PKM2 was required for tumor progression of glioma, and PKM2 might be used in a high energy production process in tumorigenesis. There were no differences between males and females for PKM2 overexpression in glioma cells. This suggested that gender did not affect PKM2 overexpression in tumorigenesis in glioma. Since some glioma tissues seemed to overexpress PKM2 more highly than others, it is important to investigate the genetic characteristics of patients with highly overexpressed PKM2 in the future.

A previous study has reported that elevated PKM2 expression is dependent on the tumor grade (34). However, we did not detect a correlation between the expression of PKM2 and grade-dependency. Race or age differences among glioma tissues might result in glioma gradeindependent results in our PKM2 overexpression studies. Interestingly, although we did not find grade-dependence of PKM2 expression, we did find that adjacent normal tissues in glioma exhibited increased expression of PKM2 when compared with normal tissues, although the expression levels were lower than in glioma tissues. This suggested that overexpression of PKM2 in adjacent tissues occurs during the tumorigenesis. We hypothesize that the phenotypes of adjacent glioma tissues were normal, but they had genetic alterations in tumorigenesis, with an increased PKM2 level. PKM2 overexpression is assumed to be an important factor in the development of tumorigenesis. More investigations should be conducted to determine whether PKM2 overexpression may be a bio-indicator for the early stages of tumorigenesis in glioma.

Furthermore, although few studies have investigated PKM2 inhibition in glioma tumorigenesis, it remains unclear whether PKM2 inhibitors may be potential glioma-targeting drugs. Several PKM2 inhibitors, such as shikonin, metformin, vitamin $\mathrm{K}$, and temozolomide, have been studied $(25,26)$. Recently, the biological effects of novel naphthoquinone derivatives have been reported as selective inhibitors of PKM2. Some compounds, such as compound $3 \mathrm{~K}$, have shown significant antiproliferative activity in various cancer cell lines with high expression of PKM2, and have exhibited more potent PKM2 inhibition than the conventional PKM2 inhibitor shikonin (12, 28-31). Here, we evaluated and aimed to identify novel findings for the anticancer effects of PKM2 inhibitors in the glioma cell line U87MG. We found that they inhibited U87MG growth at a relatively low dose. Interestingly, we found that the effect of shikonin was dependent on cultures having a low confluence, suggesting that PKM2 inhibitors may not work in a high cell-density state. It is possible that cancer cells do not use PKM2 activity for their growth in high-density states. This finding may help in further clinical applications of PKM2 inhibitors in glioma therapy.

A detailed analysis of the mechanisms of action was performed for PKM2 inhibitors shikonin and compound $3 \mathrm{~K}$. They showed these agents highly increased the level of lateapoptosis, as determined by annexin $\mathrm{V}$ analysis, whereas vincristine treatment increased early apoptosis. We also confirmed that the increase in late-apoptosis was due to increased condensation with shikonin treatment compared to vincristine; this suggested that PKM2 inhibitors more rapidly increased apoptosis than did vincristine.

Overexpression of PKM2 has been shown to increase glucose uptake, lactate production, and autophagy inhibition, thereby accelerating oncogenic growth (25). We found that shikonin increased autophagy, suggesting that PKM2 in glioma has a functional role in reducing autophagy for cancer cell survival. Vincristine increases $\mathrm{G}_{2}$-phase arrest by microtubule targeting; we accordingly observed an increase in $\mathrm{G}_{2}$-phase arrest after vincristine treatment. However, we did not detect increased $\mathrm{G}_{2}$-phase arrest after treatment with PKM2 inhibitor, although the proportion of S-phase cells slightly increased. This suggests that PKM2 inhibitors increase late apoptosis faster than cell-cycle arrest. We assumed that there was insufficient time for glioma cells to recover from the effects of PKM2 inhibitors, whereas with vincristine, they had recovery time with $\mathrm{G}_{2}$-phase arrest, leading to less late apoptosis. We conclude that the rapid increases in apoptosis and late-apoptosis levels in a relatively short time by PKM2 inhibitors resulted from non- $\mathrm{G}_{2}$-phase arrest and increased induction of autophagy.

Finally, we tested whether PKM2 inhibitors increased the effect of an anticancer drug in co-treatment. However, we did not find any increase in effect upon co-treatment with vincristine and either shikonin or compound $3 \mathrm{~K}$. Surprisingly, we found that co-treatment with both shikonin and compound $3 \mathrm{~K}$ increased efficacy compared to single treatments. These results suggest that PKM2 inhibition is sufficient for effects on glioma cells without the use of an anticancer drug. They also suggest that PKM2 inhibitors can be used as substitutes for anticancer drugs in glioma therapy, and supports the use of the combination of two different PKM2 inhibitors clinically. Our findings identify PKM2 inhibitors with antitumor activity, which lays the foundation for the development of PKM2 inhibitors as promising antitumor drugs. 


\section{Conflicts of Interest}

The Authors declare no conflicts of interest regarding this study.

\section{Authors' Contributions}

Jae Hyeon Park and Jin-Sol Lee: Collected the data, contributed data or analysis tools, wrote the article. Yunmoon Oh, Ji Sun Lee, Hae Eun Park, Haeun Lee, Yeon Su Park, So Young Kyung: Contributed data or analysis tools. Hyung Sik Kim and Sungpil Yoon: Contributed data or analysis tools, conceived and designed the analysis, collected the data, contributed data or analysis tools, wrote the article.

\section{Acknowledgements}

The Authors thank SU HYUN LEE for their technical support and preparation of the manuscript. This work was supported by National Research Foundation of Korea (NRF) funded by the Ministry of Education (NRF-2019R1A2C2002923).

\section{References}

1 Chen W, Lei C, Liu P, Liu Y, Guo X, Kong Z, Wang Y, Dai C, Wang Y, Ma W and Wang Y: Progress and prospects of recurrent glioma: a recent scientometric analysis of the web of science in 2019. World Neurosurg 134: e387-e399, 2020. PMID: 31639500. DOI: 10.1016/j.wneu.2019.10.078

2 Tan AC, Ashley DM, López GY, Malinzak M, Friedman HS and Khasraw M: Management of glioblastoma: State of the art and future directions. CA Cancer J Clin 70(4): 299-312, 2020. PMID: 32478924. DOI: 10.3322/caac.21613

3 Zhang X, Ding K, Wang J, Li X and Zhao P: Chemoresistance caused by the microenvironment of glioblastoma and the corresponding solutions. Biomed Pharmacother 109: 39-46, 2019. PMID: 30391707. DOI: 10.1016/j.biopha.2018.10.063

4 Basso J, Miranda A, Sousa J, Pais A and Vitorino C: Repurposing drugs for glioblastoma: From bench to bedside. Cancer Lett 428: 173-183, 2018. PMID: 29729291. DOI: 10.1016/j.canlet.2018.04.039

5 Kang X, Zheng Y, Hong W, Chen X, Li H, Huang B, Huang Z, Tang $\mathrm{H}$ and Geng W: Recent advances in immune cell therapy for glioblastoma. Front Immunol 11: 544563, 2020. PMID: 33193310. DOI: 10.3389/fimmu.2020.544563

6 Zheng X, Tang Q, Ren L, Liu J, Li W, Fu W, Wang J and Du G: A narrative review of research progress on drug therapies for glioblastoma multiforme. Ann Transl Med 9(11): 943, 2021. PMID: 34350258. DOI: 10.21037/atm-20-8017

7 Saeed H, Tseng YD and Lo SS: Narrative review of palliative hypofractionated radiotherapy for high grade glioma. Ann Palliat Med 10(1): 846-862, 2021. PMID: 33040565. DOI: 10.21037/ apm-20-1246

8 Chen XS, Li LY, Guan YD, Yang JM and Cheng Y: Anticancer strategies based on the metabolic profile of tumor cells: therapeutic targeting of the Warburg effect. Acta Pharmacol Sin 37(8): 1013-1019, 2016. PMID: 27374491. DOI: 10.1038/aps. 2016.47

9 Dey P, Kundu A, Sachan R, Park JH, Ahn MY, Yoon K, Lee J, Kim ND, Kim IS, Lee BM and Kim HS: PKM2 knockdown induces autophagic cell death via AKT/mTOR pathway in human prostate cancer cells. Cell Physiol Biochem 52(6): 15351552, 2019. PMID: 31135122. DOI: $10.33594 / 000000107$

10 Dey P, Son JY, Kundu A, Kim KS, Lee Y, Yoon K, Yoon S, Lee BM, Nam KT and Kim HS: Knockdown of pyruvate kinase M2 inhibits cell proliferation, metabolism, and migration in renal cell carcinoma. Int J Mol Sci 20(22): 5622, 2019. PMID: 31717694. DOI: 10.3390/ijms20225622

11 Liu VM and Vander Heiden MG: The role of pyruvate kinase M2 in cancer metabolism. Brain Pathol 25(6): 781-783, 2015. PMID: 26526946. DOI: 10.1111/bpa.12311

12 Park JH, Kundu A, Lee SH, Jiang C, Lee SH, Kim YS, Kyung SY, Park SH and Kim HS: Specific pyruvate kinase M2 inhibitor, compound $3 \mathrm{~K}$, induces autophagic cell death through disruption of the glycolysis pathway in ovarian cancer cells. Int J Biol Sci 17(8): 1895-1908, 2021. PMID: 34131394. DOI: 10.7150/ijbs.59855

13 Ni W, Xia Y, Luo L, Wen F, Hu D, Bi Y and Qi J: High expression of ALDH1A3 might independently influence poor progressionfree and overall survival in patients with glioma via maintaining glucose uptake and lactate production. Cell Biol Int 44(2): 569582, 2020. PMID: 31642564. DOI: 10.1002/cbin.11257

14 Yang W and Lu Z: Pyruvate kinase M2 at a glance. J Cell Sci 128(9): 1655-1660, 2015. PMID: 25770102. DOI: 10.1242/ jcs. 166629

15 Zhu H, Luo H, Zhu X, Hu X, Zheng L and Zhu X: Pyruvate kinase M2 (PKM2) expression correlates with prognosis in solid cancers: a meta-analysis. Oncotarget 8(1): 1628-1640, 2017. PMID: 27911861. DOI: 10.18632/oncotarget.13703

16 Tamada M, Suematsu M and Saya H: Pyruvate kinase M2: multiple faces for conferring benefits on cancer cells. Clin Cancer Res 18(20): 5554-5561, 2012. PMID: 23071357. DOI: 10.1158/1078-0432.CCR-12-0859

17 Chen WJ, Chen LH, Wang J, Wang ZT, Wu CY, Sun K, Ding BY, Liu N and Xu RX: LHPP impedes energy metabolism by inducing ubiquitin-mediated degradation of PKM2 in glioblastoma. Am J Cancer Res 11(4): 1369-1390, 2021. PMID: 33948363.

18 Lu B, Wang Z, Ding Y, Wang X, Lu S, Wang C, He C, Piao M, Chi G, Luo Y and Ge P: RIP1 and RIP3 contribute to shikonininduced glycolysis suppression in glioma cells via increase of intracellular hydrogen peroxide. Cancer Lett 425: 31-42, 2018. PMID: 29608987. DOI: 10.1016/j.canlet.2018.03.046

19 Jiang C, Lee SH, Park JH, Lee JS, Park JW, Kim JR, Lee SH, Kim HS and Yoon S: A low dose of aripiprazole has the strongest sensitization effect among 19 repositioned bipolar drugs in P-gp-overexpressing drug-resistant cancer cells. Anticancer Res 41(2): 687-697, 2021. PMID: 33517273. DOI: 10.21873/anticanres.14820

20 Kim JY, Park YJ, Lee BM and Yoon S: Co-treatment with HIV protease inhibitor nelfinavir greatly increases late-phase apoptosis of drug-resistant KBV20C cancer cells independently of P-glycoprotein inhibition. Anticancer Res 39(7): 3757-3765, 2019. PMID: 31262902 . DOI: 10.21873/anticanres.13524

21 Kim KS, Jiang C, Kim JY, Park JH, Kim HR, Lee SH, Kim HS and Yoon S: Low-dose crizotinib, a tyrosine kinase inhibitor, highly and specifically sensitizes P-glycoprotein-overexpressing chemoresistant cancer cells through induction of late apoptosis in vivo and in vitro. Front Oncol 10: 696, 2020. PMID: 32528877. DOI: 10.3389 /fonc. 2020.00696 
22 Kim JY, Kim KS, Kim IS and Yoon S: Histamine receptor antagonists, loratadine and azelastine, sensitize P-gpoverexpressing antimitotic drug-resistant KBV20C cells through different molecular mechanisms. Anticancer Res 39(7): 3767 3775, 2019. PMID: 31262903. DOI: 10.21873/anticanres.13525

23 Kim JY, Son JY, Lee BM, Kim HS and Yoon S: Aging-related repositioned drugs, donepezil and sildenafil citrate, increase apoptosis of anti-mitotic drug-resistant KBV20C cells through different molecular mechanisms. Anticancer Res 38(9): 51495157, 2018. PMID: 30194162. DOI: 10.21873/anticanres.12837

24 Vatter T, Klumpp L, Ganser K, Stransky N, Zips D, Eckert F and Huber SM: Against repurposing methadone for glioblastoma therapy. Biomolecules 10(6): 917, 2020. PMID: 32560384. DOI: 10.3390/biom 10060917

25 Alquraishi M, Puckett DL, Alani DS, Humidat AS, Frankel VD, Donohoe DR, Whelan J and Bettaieb A: Pyruvate kinase M2: A simple molecule with complex functions. Free Radic Biol Med 143: 176-192, 2019. PMID: 31401304. DOI: 10.1016/ j.freeradbiomed 2019.08 .007

26 Guo C, He J, Song X, Tan L, Wang M, Jiang P, Li Y, Cao Z and Peng C: Pharmacological properties and derivatives of shikoninA review in recent years. Pharmacol Res 149: 104463, 2019. PMID: 31553936. DOI: 10.1016/j.phrs.2019.104463

27 Kim JH, Kim TY, Kim HS, Hong S and Yoon S: Lower salinomycin concentration increases apoptotic detachment in high-density cancer cells. Int J Mol Sci 13(10): 13169-13182, 2012. PMID: 23202945. DOI: 10.3390/ijms 131013169

28 Ning X, Qi H, Li R, Jin Y, McNutt MA and Yin Y: Synthesis and antitumor activity of novel 2, 3-didithiocarbamate substituted naphthoquinones as inhibitors of pyruvate kinase M2 isoform. $\mathrm{J}$ Enzyme Inhib Med Chem 33(1): 126-129, 2018. PMID: 29185365. DOI: 10.1080/14756366.2017.1404591

29 Ning X, Qi H, Li R, Li Y, Jin Y, McNutt MA, Liu J and Yin Y: Discovery of novel naphthoquinone derivatives as inhibitors of the tumor cell specific M2 isoform of pyruvate kinase. Eur J Med Chem 138: 343-352, 2017. PMID: 28688274. DOI: 10.1016/j.ejmech.2017.06.064
$30 \mathrm{Xu} \mathrm{F}$, Guo M, Huang W, Feng L, Zhu J, Luo K, Gao J, Zheng $\mathrm{B}$, Kong LD, Pang T, Wu X and Xu Q: Annexin A5 regulates hepatic macrophage polarization via directly targeting PKM2 and ameliorates NASH. Redox Biol 36: 101634, 2020. PMID: 32863213. DOI: 10.1016/j.redox.2020.101634

31 Zeng Z, Lan J, Lei S, Yang Y, He Z, Xue Y and Chen T: Simultaneous inhibition of ornithine decarboxylase 1 and pyruvate kinase M2 exerts synergistic effects against hepatocellular carcinoma cells. Onco Targets Ther 13: 1169711709, 2020. PMID: 33244237. DOI: 10.2147/OTT.S240535

32 Morfouace M, Lalier L, Oliver L, Cheray M, Pecqueur C, Cartron PF and Vallette FM: Control of glioma cell death and differentiation by PKM2-Oct4 interaction. Cell Death Dis 5: e1036, 2014. PMID: 24481450. DOI: 10.1038/cddis.2013.561

33 Wang X, Lu S, He C, Wang C, Wang L, Piao M, Chi G, Luo Y and Ge P: RSL3 induced autophagic death in glioma cells via causing glycolysis dysfunction. Biochem Biophys Res Commun 518(3): 590-597, 2019. PMID: 31445705. DOI: 10.1016/j.bbrc. 2019.08 .096

34 Mukherjee J, Phillips JJ, Zheng S, Wiencke J, Ronen SM and Pieper RO: Pyruvate kinase M2 expression, but not pyruvate kinase activity, is up-regulated in a grade-specific manner in human glioma. PLoS One 8(2): e57610, 2013. PMID: 23451252. DOI: $10.1371 /$ journal.pone.0057610
Received October 6, 2021

Revised December 8, 2021

Accepted December 10, 2021 